\title{
Procesos avanzados de oxidación basados en ozono como alternativa de tratamiento para lixiviados de rellenos sanitarios
}

\section{Advanced Oxidation Processes Based on Ozone as a Treatment Alternative for Landfill Leaches}

\author{
Dorance Becerra-Moreno ${ }^{1,2}$, Yrany Rubio-Gomez ${ }^{1}$, Luisa Fernanda Ramírez-Ríos ${ }^{1}$, Andrés Fernando \\ Barajas-Solano $^{1}$ y Fiderman Machuca-Martínez ${ }^{2,3}$
}

\section{Resumen}

Los procesos basados en ozono hacen parte de los Procesos Avanzados de Oxidación que se han aplicado sobre los lixiviados de rellenos sanitarios. Las características altamente tóxicas de los lixiviados de rellenos sanitarios, pueden reducirse por medio de los procesos basados en ozono, los cuales han demostrado alcanzar altas remociones de contaminantes, en especial, de compuestos orgánicos recalcitrantes. En esta revisión se compila información reciente (2015 a 2021) referente a los procesos basados en ozono, donde se ha estudiado la eficacia como tratamiento, se analiza las condiciones óptimas de acuerdo al tipo de lixiviado y al método de tratamiento a emplear. Se identifica la tendencia de implementación como pretratamiento y postratamiento acoplado a procesos convencionales.

Palabras clave: lixiviado de rellenos sanitarios, ozono, PAOs, tratamiento

\begin{abstract}
Ozone-based processes are part of the Advanced Oxidation Processes that have been applied on leachates from landfills. The highly toxic characteristics of landfill leachates can be reduced by ozone-based processes, which have been shown to achieve high removals of pollutants, especially recalcitrant organic compounds. In this review, recent information is compiled (2015 to 2021) regarding ozone-based processes, where the efficacy as a treatment has been studied, the optimal conditions are analyzed according to the type of leachate and the treatment method to be used. The implementation trend is identified as pre-treatment and post-treatment coupled with conventional processes.
\end{abstract}

Keywords: landfill leachate, ozone, AOPs, treatment

Recepción: 20-feb-2021

Aceptación: 27-May-2021

\footnotetext{
${ }^{1}$ Departamento de Ciencias del Medio Ambiente, Facultad de Ciencias Agrarias y del Ambiente, Universidad Francisco de Paula Santander, Cúcuta, Colombia.

${ }^{2}$ Escuela de Ingeniería Química, Facultad de Ingeniería, Universidad del Valle, Cali Colombia.

${ }^{3}$ CENM, Facultad de Ingeniería, Universidad del Valle. Cali, Colombia.

Autor de correspondencia: fiderman.machuca@correounivalle.edu.co
} 


\section{Introducción}

La generación de Residuos Sólidos Urbanos (RSU) va en aumento y los rellenos sanitarios son el método generalmente implementado para disponerlos de manera controlada [1]. Los rellenos sanitarios han sido considerados como una alternativa de destino final para los residuos sólidos, por ser de fácil operación, viables económicamente, y ambientalmente aceptables si son gestionados de forma adecuada [2, 3, 4].

Un aspecto negativo de la operación de los rellenos sanitarios son los lixiviados, los cuales son líquidos de color obscuro altamente contaminantes [5]. Éstos se producen principalmente a partir de la degradación de los residuos, tanto orgánicos como inorgánicos, y la percolación de agua de lluvia [6, 7, 8]. Youcai (2018) describe la formación de lixiviados en un relleno sanitario típico, lo cual consiste generalmente en el ingreso de agua lluvia a la zona de residuos en fase sólida, transportando los contaminantes solubles a la fase líquida. Al mismo tiempo, los residuos orgánicos se descomponen en materias orgánicas solubles (como los ácidos grasos volátiles). La humedad de los residuos, la acción de los microorganismos, entre otras condiciones, propician la degradación mediante una serie de procesos físicos, químicos y biológicos complejos [8]. En la Figura 1 se presenta un esquema sobre la producción de lixiviados a partir de residuos sólidos de rellenos sanitarios.

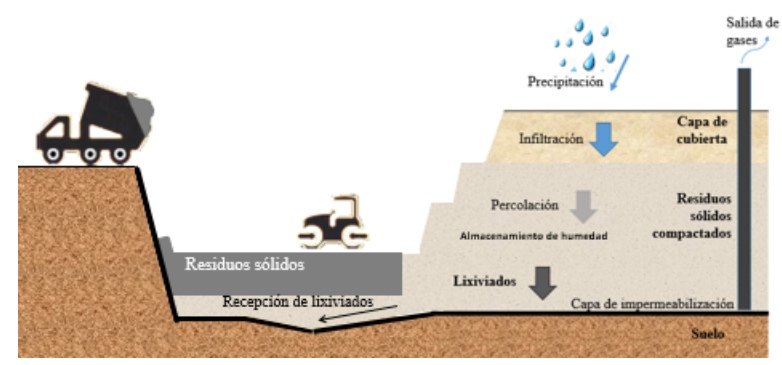

Figura 1. Producción de lixiviados en un relleno sanitario.

Las características contaminantes de los lixiviados se ven influenciadas particularmente por la edad del relleno sanitario [9], es decir, los lixiviados de rellenos sanitarios jóvenes ( $<5$ años) contienen altas cantidades de contaminantes orgánicos y presentan un pH ácido [10]. Mientras los lixiviados de rellenos sanitarios de edad intermedia (5-10 años) y los maduros ( $>10$ años), se caracterizan por presentar alto contenido de compuestos húmicos y fulvicos, así como, menor biodegradabilidad en comparación con los rellenos sanitarios jóvenes [11]. Además, estos lixiviados presentan contaminantes como metales pesados, compuestos xenobióticos, altas concentraciones de amonio, entre otras sustancias tóxicas, perjudiciales tanto para el ambiente como la salud pública [12].

Debido a las características de los lixiviados de rellenos sanitarios, presentan altas concentraciones en, Demanda Química de Oxígeno (DQO), Demanda Biológica de Oxígeno (DBO), Carbono Orgánico Disuelto (COD), Carbono Orgánico Total (COT), compuestos absorbentes de $\mathrm{UV}_{254}\left(\mathrm{UV}_{254}\right)$ y nitrógeno amoniacal $\left(\mathrm{NH}_{3}-\mathrm{N}\right)$, así también, una baja proporción de DBO/DQO (relación de biodegradabilidad) [13, 14]. En consecuencia, el tratamiento de los lixiviados es relevante dentro de los rellenos sanitarios, donde se deben incluir métodos que reduzcan las concentraciones de los contaminantes y disminuyan sus cualidades nocivas. Existen varios métodos de tratamiento como lo son: físicos (recirculación y evaporación) [14], biológicos (lodos activados, reactores con biopelículas sumergidas y fijas, reactores de manto de lodos de flujo ascendente) [15], tecnologías de membrana (microfiltración, ultrafiltración y osmosis inversa) y Procesos Avanzados de Oxidación (Procesos foto-asistido, procesos electroquímicos, procesos varios y procesos basados en ozono) [16].

Los Procesos Avanzados de Oxidación (PAOs) han sido utilizados como métodos de tratamiento de lixiviados por su ventaja para degradar compuestos recalcitrantes, superando a algunos de los demás tratamientos [17]. Principalmente, los PAOs presentan una alta eficacia de eliminación de compuestos orgánicos y aumentan la biodegradabilidad [18, 19]. Uno de los tratamientos PAOs más comunes son los procesos basados en ozono, los cuales presentan su alto valor oxidante para producir radicales hidroxilos $(\mathrm{OH})$ que degradan los contaminantes orgánicos recalcitrantes [18, 2]. Asimismo, los procesos basados en ozono son tratamientos ventajosos en cuanto a la 
remoción de color, olor y algunos metales pesados [20].

El presente trabajo es una revisión centrada en el desarrollo de los procesos basados en ozono para el tratamiento de lixiviados producidos en rellenos sanitarios, como un método de los PAOs que ha presentado una buena eficacia en la remoción de la concentración de los contaminantes. Se realiza un análisis descriptivo y comparativo de las investigaciones científicas recientes, entre los años 2015 y 2021, en el tema de los procesos basados en ozono para tratar lixiviados. Así mismo se citan referencias para profundizar y ampliar la información actualizada.

Las investigaciones consideradas para la presente revisión, se extrajeron de buscadores como Science Direct, Springer Link, Taylor \& Francis, entre otros. De estas investigaciones, el $68 \%$ pertenecen a categoría Q1, el 29\% a categoría Q2 y solo el 3\% a categoría Q3. Las revistas científicas que mayor cantidad de publicaciones registran sobre el tema de estudio, según esta revisión son: Journal of Cleaner Production, Waste Management, Process Safety and Environmental Protection, Desalination and Water Treatment, Water Research, Chemosphere, Chemical Engineering Journal, y Environmental Science and Pollution Research. En cuanto a la distribución de las investigaciones según el año de publicación, al 2015 pertenece el $12 \%$ del total de las referencias bibliográficas, 2016 el 14\%, 2017 el 12\%, 2018 el 10\%, 2019 el 20\%, 2020 el 29\%; y al año 2021 corresponde el $3 \%$ de las publicaciones científicas revisadas hasta el mes de febrero.

\section{PAOs como tratamiento}

Los PAOs han sido estudiados en múltiples investigaciones para demostrar la eficiencia de su aplicación como tratamiento en líquidos con características de alta toxicidad (aguas residuales y los lixiviados). La velocidad del tratamiento, la mejora de la biodegradabilidad y el alto porcentaje de remoción de contaminantes orgánicos recalcitrantes, posicionan a los PAOs como un método de tratamiento ventajoso frente a otros (físicos y biológicos entre los más comunes). Según Kow et al. (2016), son dos los procesos que se desarrollan dentro del tratamiento mediante los PAOs. Uno es la formación de radicales libres u oxidantes como radicales hidroxilos $(\mathrm{OH})$ y otras especies [21]. Otro proceso es la reacción química de radicales producidos con compuestos orgánicos, lo cual se representa como reacción básica en la ecuación (1) [3].

$$
\begin{aligned}
& \text { Oxidante }+ \text { Compuesto Orgánico } \rightarrow \\
& \text { Subproductos de Oxidación } \rightarrow \mathrm{CO}_{2}+\mathrm{H}_{2} \mathrm{O}
\end{aligned}
$$

Los PAOs como tratamiento, se les han atribuido un alto valor en términos de costo, por lo tanto, se considera que su implementación es más favorable como pretratamiento o postratamiento, que como tratamiento único [15, 22]. Por consiguiente, generalmente para el tratamiento de lixiviados de rellenos se opta por crear un sistema usando a los PAOs como complemento de uno o más métodos acoplados estratégicamente hasta alcanzar las remociones de contaminantes esperadas. Una de las combinaciones de métodos de tratamiento de lixiviados más usadas junto con los PAOs, son los tratamientos biológicos, por sus buenos resultados de eficacia de remoción en cuanto DQO, entre otros parámetros [7].

Se ha afirmado que el aumento del rendimiento de los PAOs se logra con la manipulación de las variables asociadas al tipo de oxidante (Radical hidroxilo, $\mathrm{OH}$; radical sulfato, $\mathrm{SO}_{4}^{-}$; ozono, $\mathrm{O}_{3}$; anión persulfato, $\mathrm{S}_{2} \mathrm{O}_{8}{ }^{2-}$; peróxido de hidrógeno, $\mathrm{H}_{2} \mathrm{O}_{2}$; radical hidroperóxido, $\mathrm{HO}_{2} ;$ y radical superóxido, $\mathrm{O}_{2}{ }^{-}$) y sus concentraciones, de lo cual, la información del potencial de oxidación $\mathrm{E}^{0}(\mathrm{~V})$ y su funcionamiento dentro del tratamiento es compilada y presentada por Kow et al. (2016). Además, las pruebas realizadas a diferentes PAOs y otros métodos como el biológico, coagulación-floculación y demás, para determinar los rendimientos como tratamiento de lixiviados de un relleno sanitario, se encuentran en la investigación de Bashir et al. (2015).

\section{Procesos basados en ozono como tratamiento de lixiviados}

Los procesos basados en ozono se han catalogado como una de las categorías de los PAOs más 
prometedoras para eliminar y/o reducir contaminantes de los lixiviados a estándares permisibles expedidos las autoridades ambientales [3]. Los procesos basados en ozono se muestran en la Figura 2, elaborada con base en los estudios de Ikehata $\&$ Li (2018) y Kow et al. (2016). Recientemente, se han documentado excelentes resultados de estos procesos, especialmente como pretratamiento y postratamiento.

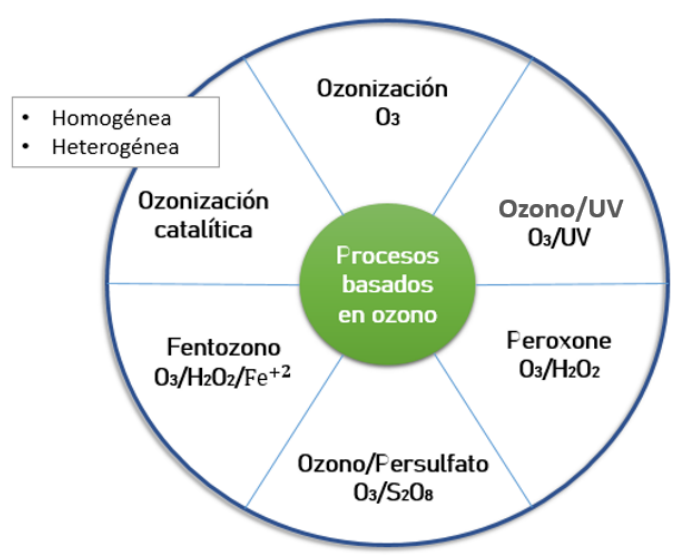

Figura 2. Tratamientos de procesos basados en ozono.

El rendimiento como pretratamiento se le confiere al favorable aumento de la biodegradabilidad y reducción de la toxicidad de compuestos orgánicos $[4,24]$. Por ejemplo, un pretratamiento con $\mathrm{O}_{3}$ para tratar lixiviados en membranas de alta presión (osmosis inversa y nanofiltración), obtuvo una reducción de $55.5 \%$ de compuestos absorbentes de DQO y 54.4\% de COD [4]. El proceso de $\mathrm{O}_{3}$ puede exitosamente eliminar y convertir compuestos de ácido húmico y ácido Fulvico en fracciones hidrófilas que son biodegradables [4].

Como postratamiento, los procesos basados en ozono cumplen con la función de oxidar los compuestos orgánicos residuales del tratamiento anterior a este. Por ejemplo, el proceso con ozono/persulfato $\left(\mathrm{O}_{3} / \mathrm{S}_{2} \mathrm{O}_{8}\right)$ como postratamiento, se aplicó a un lixiviado tratado mediante un proceso biológico, obtuvo remociones de $87 \%$ de DQO y $85 \%$ de color, así como un aumento de la relación de biodegradabilidad de 0.13 a 0.61 [1]. A su vez, los procesos basados en ozono son ventajosos como método de tratamiento limpio, puesto que regularmente la generación de lodos es baja o poco significativa [26, 27], a excepción de los procesos asociados a iones metálicos [14].

Las reacciones químicas involucradas en cada proceso basado en ozono (ozonización, ozonización con radiación UV, peroxono, ozono/persulfato, fentozono, ozonización catalítica) están disponibles en el trabajo de Kow et al. (2016) y el libro de Ikehata \& Li (2018).

\subsection{Ozonización $\left(\mathrm{O}_{3}\right)$}

La oxidación es el proceso principal de la ozonización para degradar y mineralizar contaminantes orgánicos de gran tamaño, de líquidos como los lixiviados [14], en especial los aromáticos [10]. Los compuestos aromáticos se relacionan con los compuestos absorbentes $\left(\mathrm{UV}_{254}\right)$, a lo que las pruebas de tratamiento con $\mathrm{O}_{3}$ han demostrado ser altamente eficaces $[10,25]$.

La ozonización degrada los compuestos orgánicos recalcitrantes en el lixiviado de vertederos mediante reacciones directas o indirectas. La reacción directa es el mecanismo en el cual se produce una reacción redox, entre las moléculas de ozono y la materia orgánica del lixiviado del vertedero. En cuanto al mecanismo mediante la reacción indirecta, se producen radicales $\mathrm{OH}$ altamente activos durante la oxidación, que luego inducen una reacción en cadena, que implican reacciones de iniciación, propagación y terminación [23]. El radical $\mathrm{OH}$ de alto potencial redox, interactúa con los contaminantes orgánicos del lixiviado, lo que propicia la apertura del anillo en compuestos orgánicos tóxicos, mejorando así la biodegradabilidad. La reacción indirecta es considera más eficiente en comparación con la reacción directa [3].

$\mathrm{El} \mathrm{O}_{3}$ como tratamiento de lixiviados remueve los contaminantes de formas distintas, de acuerdo a las condiciones de $\mathrm{pH}$ [3]. Las reacciones de iniciación principales se presentan en la ecuación(2) para un $\mathrm{pH}$ acido a neutro y las Ecuaciones (3) y (4) para un $\mathrm{pH}$ alcalino [23].

$$
\begin{aligned}
& \mathrm{O}_{3}+\mathrm{OH}^{-} \rightarrow \mathrm{HO}_{2}+\mathrm{O}_{2}^{-} \\
& \mathrm{O}_{3}+\mathrm{OH}^{-} \rightarrow \mathrm{HO}_{2}^{-}+\mathrm{O}_{2}
\end{aligned}
$$




$$
\mathrm{O}_{3}+\mathrm{HO}_{2}^{-} \rightarrow \mathrm{HO}_{2}+\mathrm{O}_{3}^{-}
$$

A pH ácidos, la remoción de contaminantes ocurre de forma directa por medio del proceso de reacción química con los compuestos orgánicos, por ejemplo, Kattel et al. (2016) obtuvieron remociones aproximadas del $45 \%$ de $\mathrm{DBO}_{7}$ y $18 \%$ de toxicidad en medio ácido. En contraste, en este tratamiento se registraron remociones aproximadas del $65 \%$ de $\mathrm{DBO}_{7}$ y $23 \%$ de toxicidad en medio alcalino, debido a la oxidación indirecta de los $\mathrm{OH}$.

Huawei et al. (2016) por su parte, al tratar lixiviados efluentes de los tratamientos con osmosis inversa y nanofiltración, en medio alcalino, encontraron que se producían mayores valores de $\mathrm{NH}_{3}-\mathrm{N}$ y $\mathrm{NO}_{3}-\mathrm{N}$ después de la ozonización. Según explican los autores, esto es debido a la degradación de los grupos amino en las sustancias húmicas o la degradación de otros compuestos orgánicos que contienen nitrógeno. Por el contrario, un medio alcalino inicial es favorable en la remoción de contaminantes como el color y los compuestos absorbentes $\mathrm{UV}_{254}$, por medio de procesos de generación de radicales $\mathrm{OH}$ u otras especies. Esto es representado en los estudios de, AlGburi et al. (2020) quienes alcanzaron una remoción de color de hasta $90 \%$ y, Soubh \& Mokhtarani (2016) que obtuvieron en sus resultados una remoción de $90 \%$ de compuestos absorbentes $\mathrm{UV}_{254}$. La Tabla 1 describe los parámetros iniciales, las condiciones óptimas de operación y la eficacia de los tratamientos realizados con $\mathrm{O}_{3}$ a lixiviados de rellenos sanitarios.

\subsection{Ozono/UV $\left(\mathrm{O}_{3} / \mathrm{UV}\right)$}

La ozonización en presencia de irradiación ultravioleta, con una longitud de onda $(\lambda)$ inferior a $300 \mathrm{~nm}$, es el principio para degradar los contaminantes [24]. En este sistema, el $\mathrm{O}_{3}$ se activa al absorber luz ultravioleta a $254 \mathrm{~nm}$ y se somete a fotólisis, aumentando la producción de $\mathrm{H}_{2} \mathrm{O}_{2}$, y éste también se someterán a fotólisis para producir OH [29]. Básicamente, el mecanismo del proceso de tratamiento $\mathrm{O}_{3} / \mathrm{UV}$ es similar al de ozonización más la integración de fotones [3], como se representan en las ecuaciones (5) y 6 , reacciones de iniciación principales [23].

$$
\mathrm{O}_{3}+\mathrm{H}_{2} \mathrm{O}+\mathrm{hv} \rightarrow \mathrm{H}_{2} \mathrm{O}_{2}
$$

$$
\mathrm{H}_{2} \mathrm{O}_{2}+\mathrm{hv} \rightarrow 2 \mathrm{OH}
$$

Los rayos UV actúan como catalizadores, oxidando los contaminantes, rompiendo los enlaces saturados complejos para degradarlos, aprovechando el alto potencial de absorción de luz del ozono [30]. El rendimiento de eliminación de los contaminantes con el tratamiento $\mathrm{O}_{3} / \mathrm{UV}$, ha demostrado ser mayor a $\mathrm{pH}$ de 9 [29, 30, 31]. El $\mathrm{O}_{3} / \mathrm{UV}$ aumenta la biodegradabilidad en hasta un $91 \%$ [29], es eficaz en contaminantes refractarios, así como en sustancias húmicas y fulvicas, convirtiéndolas en intermedios alifáticos de bajo peso molecular [31]. Gomes et al. (2020) afirmaron que el rendimiento de este tratamiento para lixiviados puede estar relacionado con el diseño del fotorreactor, el cual debe asegurar que se permita un tiempo de contacto prolongado entre las moléculas de $\mathrm{O}_{3}$ y los fotones UV para obtener mayores remociones. Los estudios asociados a la aplicación de procesos de $\mathrm{O}_{3} / \mathrm{UV}$, para tratar lixiviados de rellenos sanitarios, se presentan en la Tabla 2.

\section{Peroxono $\left(\mathrm{O}_{3} / \mathrm{H}_{2} \mathrm{O}_{2}\right)$}

El Peroxono es una combinación de ozonización con la adición de oxidante fuerte, $\mathrm{H}_{2} \mathrm{O}_{2}$, lo cual mejora la transformación de $\mathrm{O}_{3}$ en $\mathrm{OH}[33,34]$. Se han realizado varios ensayos con $\mathrm{O}_{3} / \mathrm{H}_{2} \mathrm{O}_{2}$, como postratamiento de lixiviados tratados por procesos biológicos y con membrana $[24,35,36]$. Los resultados demuestran que este tratamiento puede degradar compuestos aromáticos a pH bajos [30, 35].

El mecanismo del proceso de tratamiento con $\mathrm{O}_{3} / \mathrm{H}_{2} \mathrm{O}_{2}$ para líquidos como los lixiviados, se compone de reacciones de iniciación y de terminación. En este, el ozono se descompone muy rápidamente en presencia de $\mathrm{H}_{2} \mathrm{O}_{2}$ y la mayoría de contaminantes se degradan por reacción indirecta con los radicales $\mathrm{OH}$. A continuación, se presentan las ecuaciones (7) y (8) de las reacciones de iniciación y la ecuación (9) de la reacción de terminación [3].

$$
\begin{gathered}
\mathrm{H}_{2} \mathrm{O}_{2} \leftrightarrow \mathrm{HO}_{2}^{-}+\mathrm{H}^{+} \\
\mathrm{H}_{2} \mathrm{O}_{2}+\mathrm{H}_{2} \mathrm{O} \rightarrow \mathrm{HO}_{2}^{-}+\mathrm{H}_{3} \mathrm{O}^{+} \\
\mathrm{OH}+\mathrm{HO}_{2} \rightarrow \mathrm{H}_{2} \mathrm{O}+\mathrm{O}_{2}
\end{gathered}
$$


Tabla 1. Tratamiento de lixiviados con $\mathrm{O}_{3}$

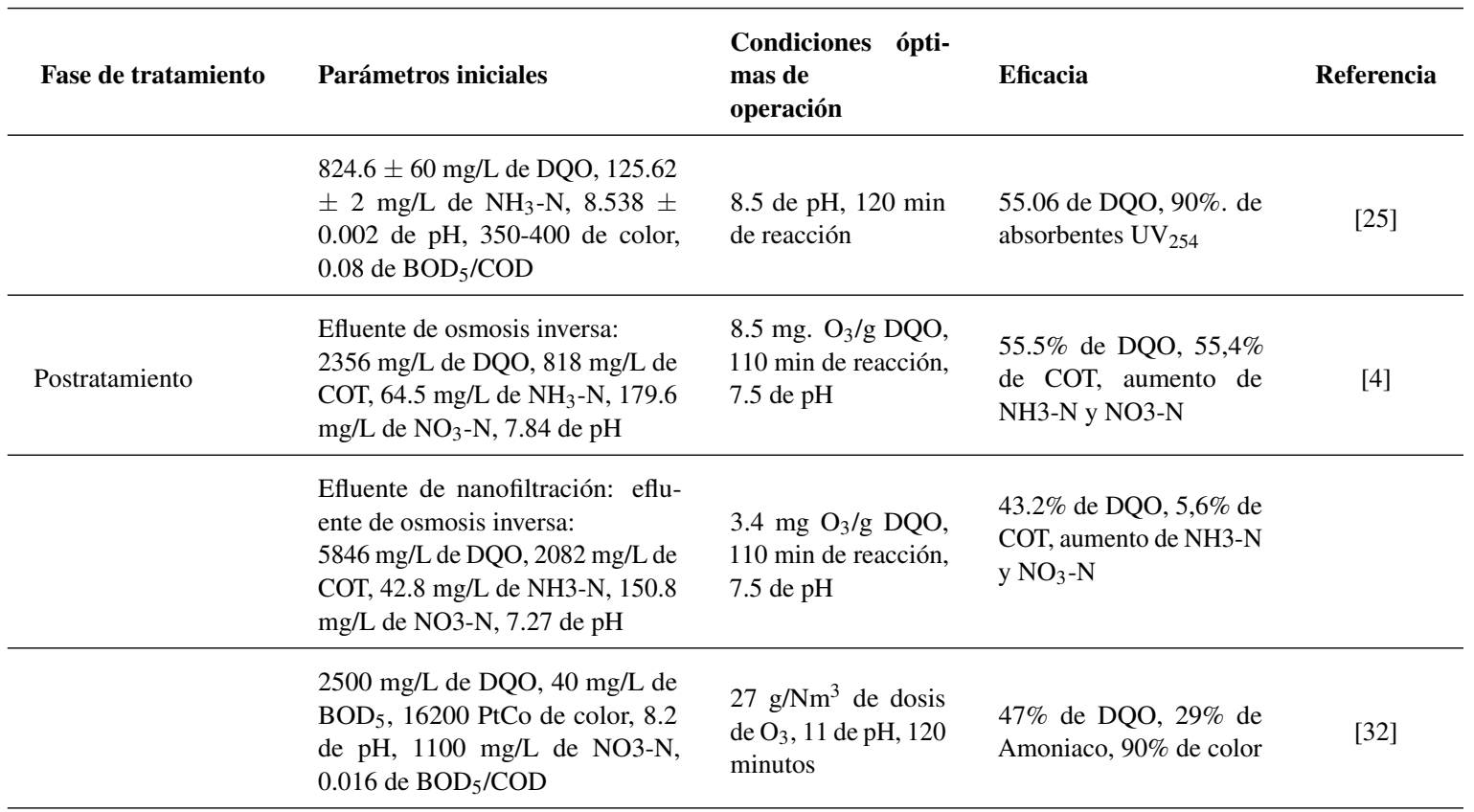

En la Tabla 2 se presentan los resultados de optimización del tratamiento con peroxono para remover contaminantes de lixiviados de rellenos sanitarios. Este tratamiento depende de la dosis de ozono aplicado para la reacción [7, 18], puesto que, en los estudios citados, las condiciones óptimas de operación se obtuvieron con la mayor dosis de $\mathrm{O}_{3}$.

\subsection{Ozono/Persulfato $\left(\mathrm{O}_{3} / \mathrm{S}_{2} \mathrm{O}_{8}{ }^{2-}\right)$}

El ozono/persulfato es una combinación de ozonización con oxidante fuerte, $\mathrm{S}_{2} \mathrm{O}_{8}{ }^{2-}$, el cual, en el proceso de descomposición puede actuar como iniciador para producir $\mathrm{SO}_{4-}$ [37]. También, se pueden producir iones $\mathrm{HSO}_{4-}$ y $\mathrm{H}_{2} \mathrm{O}_{2}$ debido a la reacción conjunta con moléculas de agua. Las reacciones en el tratamiento con radical sulfato, se basa mayormente en la transferencia de electrones, lo cual produce radicales $\mathrm{OH}$ que aceleran la depuración de la materia orgánica [22]. El mecanismo del proceso $\mathrm{O}_{3} / \mathrm{S}_{2} \mathrm{O}_{8}{ }^{2}$ se componen de reacciones principales de iniciación y propagación como se representa en las ecuaciones (10), (11) y (12) [37].

$$
\begin{gathered}
\mathrm{S}_{2} \mathrm{O}_{8}^{-2}+2 \mathrm{H}_{2} \mathrm{O} \rightarrow 2 \mathrm{HSO}_{4}^{-}+\mathrm{H}_{2} \mathrm{O}_{2} \\
\mathrm{~S}_{2} \mathrm{O}_{8}^{-2}+\mathrm{OH}^{-} \rightarrow \mathrm{SO}_{4}^{-2}+\mathrm{SO}_{4}^{-}+1 / 2 \mathrm{O}_{2}+\mathrm{H}^{+}
\end{gathered}
$$

$$
\mathrm{SO}_{4}^{-2}+\mathrm{OH}^{-} \rightarrow \mathrm{SO}_{4}^{-}+\mathrm{OH}^{-}
$$

El tratamiento con $\mathrm{O}_{3} / \mathrm{S}_{2} \mathrm{O}_{8}{ }^{2-}$ aplicado a los lixiviados, permite disminuir la toxicidad, al mismo tiempo que aumenta la biodegradabilidad, asimismo, ha sido considerado como una alternativa eficaz para la remoción simultanea de DQO y color [25]. Se asocia la condición de un pH Inicial básico para favorecer la reacción de los radicales sulfatos [22]. Soubh \& Mokhtarani (2016) optimizaron el tratamiento, a pH de 9, para obtener disminuciones en los valores de parámetros como DQO, color y toxicidad, en hasta 85,87 y $80 \%$, respectivamente, además de un aumento de $47 \%$ de $\mathrm{BOD}_{5} / \mathrm{COD}$ (Tabla 2).

Adicionalmente, se ha estudiado la implementación del tratamiento con $\mathrm{O}_{3} / \mathrm{S}_{2} \mathrm{O}_{8}{ }^{2-}$ en conjunto con un medio de activación del persulfato, con el fin de aumentar la oxidación que produce el $\mathrm{SO}_{4}{ }^{-}$[3]. Entre los medios de activación del persulfato se destacan, la activación térmica, activación por microondas, activación UV, entre otros [38]. Poblete et al. (2019) realizaron experimentos con esta alternativa para tratar lixiviados por medio de $\mathrm{UV}_{\text {solar }} / \mathrm{O}_{3} / \mathrm{S}_{2} \mathrm{O}_{8}{ }^{2-}$, aprovechando la energía del sol y el proceso de ozonización con persulfato lograron remover un $26.7 \%$ de DQO y $69.3 \%$ de color (Tabla 2). 
El tratamiento de líquidos con $\mathrm{S}_{2} \mathrm{O}_{8}{ }^{2-}$, puede atribuirse a una la elevada demanda del consumo de este oxidante [38]. Introducir una alta concentración de sulfato en el tratamiento, puede producir una serie de subproductos intermedios tóxicos. Por ejemplo, los iones sulfato y los cloruros puede dar lugar a la formación de compuestos orgánicos halogenados. En consecuencia, se inhibe la bioactividad, lo que conduce además a una baja biodegradabilidad en comparación con otros tratamientos como la ozonización única [38].

\section{Fentozono $\left(\mathrm{O}_{3} / \mathrm{H}_{2} \mathrm{O}_{2} / \mathrm{Fe}^{+2}\right)$}

Fentozono es la combinación de los procesos de ozonización y Fenton; donde, el $\mathrm{OH}$ producido en el proceso de Fenton reacciona con el $\mathrm{O}_{3}$, acelerando su descomposición. Esto conduce a una producción de mayor cantidad de radicales y por lo tanto mejora el proceso de oxidación [36], mecanismo en forma básica representado en la reacción de la ecuación (13) [3]. El mecanismo de reacción del proceso de Fenton se explica en forma detallada en el trabajo de Wu et al. (2020) [3].

$$
\mathrm{OH}+\mathrm{O}_{3} \rightarrow \mathrm{HO}_{2}+\mathrm{O}_{2} \rightarrow \mathrm{HO}_{4}
$$

En el proceso $\mathrm{O}_{3} / \mathrm{H}_{2} \mathrm{O}_{2} / \mathrm{Fe}^{+2}$ pueden generarse lodos a causa de los iones metálicos producidos a partir del $\mathrm{Fe}^{+2}$ [14]. Las investigaciones han coincidido en que el tratamiento de lixiviados con $\mathrm{O}_{3} / \mathrm{H}_{2} \mathrm{O}_{2} / \mathrm{Fe}^{+2}$ es altamente eficaz en la degradación de compuestos orgánicos recalcitrantes, como las sustancias húmicas y fúlvicas. También, los grupos funcionales se degradan y destruyen considerablemente en el medio líquido tratado [14, 36]. Las condiciones óptimas de operación que se registran en este tratamiento, se encuentran asociadas a un $\mathrm{pH}$ de 2 a 7 [35]. Asaithambi et al. (2017) obtuvieron remociones de $70.5 \%$ de DQO y $88 \%$ de color, en condiciones de $\mathrm{pH} 7.0,3.5 \mathrm{~g} / \mathrm{h}$ de dosis de $\mathrm{O}_{3}$ y $180 \mathrm{~min}$ de reacción. Por otra parte, en condiciones de $\mathrm{pH}$ ácido (3.0), Huang et al. (2019) obtuvieron los porcentajes de 48.82 y $63.59 \%$ en remoción de DQO y absorbentes $\mathrm{UV}_{254}$, respectivamente. Así mismo, Wang et al. (2021) obtuvieron remociones en condiciones óptimas de 36.45 y $54.73 \%$ de TOC y absorbentes $\mathrm{UV}_{254}$, respectivamente (Tabla 2).

\subsection{Ozonización catalítica}

Para obtener un rendimiento satisfactorio del tratamiento de ozonización de lixiviados en un menor tiempo, se han desarrollado los catalizadores. El funcionamiento de los catalizadores consiste en mejorar el poder oxidante del ozono Según el tipo de catalizador se distinguen dos grupos: homogéneos y heterogéneos [23]. Por un lado, la ozonización catalítica homogénea utiliza iones de metales de transición que descomponen el ozono mediante uno de los procesos de generación de radicales libres o la oxidación directa [39]. Entre los iones de metales en transición comunes se encuentran los nitratos, sulfatos, entre otros solubles en agua [37, 40]. La activación del ozono se da por iones como Fe(II), $\mathrm{Mn}(\mathrm{II}), \mathrm{Ni}(\mathrm{II}), \mathrm{Co}(\mathrm{II}), \mathrm{Cd}(\mathrm{II})$ o $\mathrm{Cu}(\mathrm{II})[12,14]$.

Se ha demostrado que un $\mathrm{pH}$ ácido es favorable para el tratamiento de lixiviados por ozonización catalítica homogénea [39]. Además, el tiempo de reacción es una condición importante del tratamiento, como en el estudio de Wang et al. (2020), quienes obtuvieron altas remociones de contaminantes orgánicos y metales pesados, en un tiempo de reacción de 25 minutos, a una dosis de $100 \mathrm{mg} / \mathrm{min}$ de $\mathrm{O}_{3}$ y $6 \mathrm{~g} / \mathrm{L}$ de $\mathrm{Fe}$ (III) como catalizador.

En cuanto a, la ozonización catalítica heterogénea se utilizan varios óxidos metálicos de $\mathrm{Mn}, \mathrm{Ti}, \mathrm{Al}, \mathrm{Fe}$, Si y Ce [21], así como el carbón activado [15], la zeolita natural [32] y otros materiales absorbentes de los contaminantes. Éste tratamiento hace uso de sistemas gas-liquido-solido donde el catalizador es un sólido soportado comúnmente [15, 42].

El pH básico en su mayoría, se ha caracterizado por ser la condición óptima en la ozonización catalítica heterogénea [43, 44]. Así mismo, la dosis de catalizador es una condición relevante para este tratamiento, ejemplo que lo demuestra, son los estudios realizados por He et al. (2018) y Xiang et al. (2020). Ambas investigaciones trataron lixiviados aplicando la ozonización catalizada con $\mathrm{Al}_{2} \mathrm{O}_{3}$, con similar condición inicial de DQO (1100$1317.5 \mathrm{mg} / \mathrm{L})$ y similares condiciones de operación de $\mathrm{pH}$ (7.3-7.8), dosis de ozono (18.92-20 mg/min), en un tiempo de reacción de 30 minutos. No obstante, He et al. (2018) aplicaron una dosis de $50 \mathrm{~g} / \mathrm{L}$ de $\mathrm{Al}_{2} \mathrm{O}_{3}$, con lo cual obtuvieron una remoción de $70 \%$ 
Tabla 2. Tratamiento de lixiviados con $\mathrm{O}_{3} / \mathrm{UV}, \mathrm{O}_{3} / \mathrm{H}_{2} \mathrm{O}_{2}, \mathrm{O}_{3} / \mathrm{S}_{2} \mathrm{O}_{8}{ }^{2-}, \mathrm{O}_{3} / \mathrm{H}_{2} \mathrm{O}_{2} / \mathrm{Fe}^{+2}$

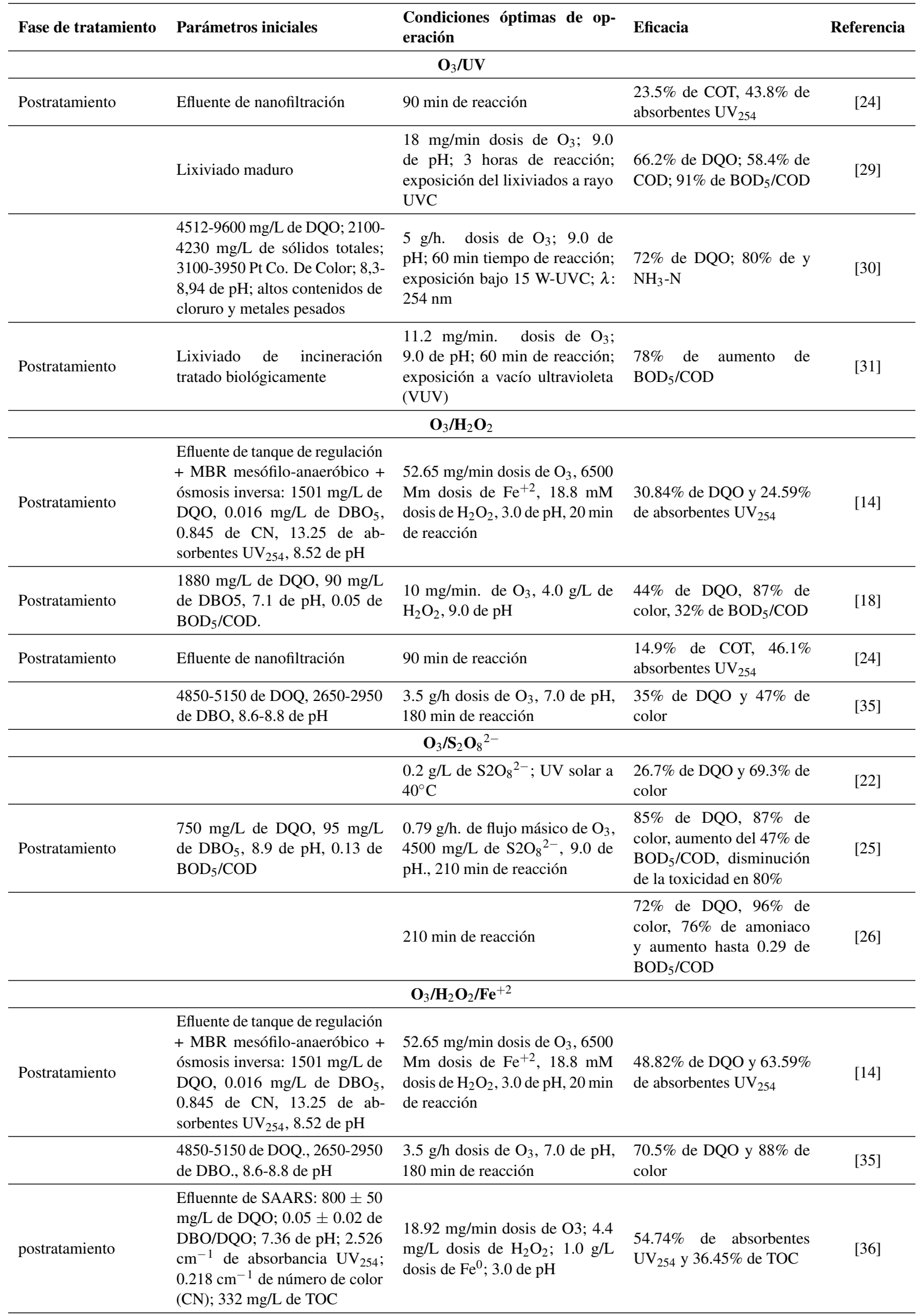


en términos de DQO, mientras Xiang et al. (2020), aplicaron una dosis de $10 \mathrm{~g} / \mathrm{L}$ de $\mathrm{Al}_{2} \mathrm{O}_{3}$ y obtuvieron una reducción menor de DQO en un 54.3\%.

En la Tabla 3 se presenta la descripción algunos de los estudios que han puesto a prueba los procesos de ozonización catalítica sobre lixiviados de rellenos sanitarios, donde se indica los resultados de remoción de contaminantes de acuerdo a las condiciones óptimas en cada caso.

\section{Desafíos en la implementación de los procesos basados en ozono a escala industrial en países en desarrollo}

Los países en desarrollo en su mayoría disponen los RSU en rellenos sanitarios o en vertederos [47]. Dentro de la gestión integrada de los rellenos sanitarios es de gran relevancia la gestión de los lixiviados, sin embargo, la mayoría no cuentan con la adecuada implementación de los procesos de tratamiento, que tengan la capacidad de manejar la cantidad y características del lixiviado generado [20]. Esto representa inminentes consecuencias para el medio ambiente y por consiguiente para la salud de la población de los países en desarrollo, lo cuales, se han visto afectados en varias ocasiones por el mal manejo de estos líquidos, que contaminan el suelo y las aguas subterráneas [48]. La gestión de lixiviados es un desafío [47, 49]; es necesario en los países en desarrollo, que los procesos de tratamiento sean altamente rentables, con un bajo requerimiento de área, simple de implementar, y mantener e identificar los factores significativos que los afectan [20, 47].

Los lixiviados están compuestos por múltiples contaminantes (altas cargas orgánicas, amonio y sales inorgánicas, así como, contenidos de hidrocarburos, pesticidas, entre otros), que lo caracterizan un líquido complejo $[1,32,44,50$, 51]. El tratamiento de los lixiviados debe alcanzar unos estándares mínimos, generalmente expedidos por la autoridad ambiental para evitar los daños ecológicos y de la salud poblacional [52]. En los países en desarrollo se han implementado los procesos convencionales físicos, biológicos y/o filtración con membrana, sin embargo, muchas veces no es suficiente [53]. Por lo tanto, es requerido aumentar la reducción de la carga contaminate por medio de métodos avanzados, como lo PAOs [20], entre los cuales, procesos basados en ozono ha sido considerados unos de los más prometedores [3].

Como ya se ha mencionado anteriormente, los procesos basados en ozono como tratamiento, son muy valorados por sus ventajas de convertir los compuestos orgánicos recalcitrantes en compuestos de menor peso molecular, aumentar la biodegradabilidad, entre otras [23]. Sin embargo, la eficiencia del tratamiento con estos procesos depende de varios factores como, los costos de tratamiento relativamente altos y la aplicación de sistemas de control [20]. Por ejemplo, el costo para el tratamiento de lixiviados por medio de ozonizacion catalizada en un reactor con microburbujas, se ha estimado que es de $5.36 \$ / \mathrm{t}$ para remover hasta un $96.25 \%$ de DQO [51]. Otra estimacion del costo realizada para el tratamiento de ozonizacion fue de $7.06 € / \mathrm{m}^{3}$, el cual es mayor, frente al costo estimado de $4.0 € / \mathrm{m}^{3}$ del tratamiento biologico oxidativo [28].

Así mismo, la aplicación exitosa de los procesos basados en ozono y en general los PAOs, está restringida por el limitado avance de los sistemas de control a escala industrial, lo cual es necesario para realizar la dosificación eficiente de acuerdo a los cambios de volumen del lixiviado a tratar, principalmente ocasionados por los periodos de lluvias [20]. Ademas, el sistema de control de ajuste del $\mathrm{pH}$ es relevante para obtener las eficiencias de remocion esperadas [12]. Cabe mencionar, los lixiviados generados en los paises en desarrollo suelen ser de $\mathrm{pH}$ neuro, en comparacion con los paises desarrollados, que generalmente, su $\mathrm{pH}$ se sitúan en el rango de los ácidos; ésta y otras caracteristicas comunes de los lixiviados generados en los paises en desarrollo se encuentran en Rajoo et al. (2020).

Las limitaciones de los procesos basados en ozono han provocado que se consideren no favorables como tratamiento único [16]. De hecho, varios estudios han demostrado que un tratamiento combinado es capaz de mejorar la calidad del lixiviado y minimizar el residuo generado a un costo de tratamiento menor que un tratamiento individual [20]. Adicionalmente, existe una complejidad de las características del lixiviado, puesto que, en ocasiones se disponen 
Tabla 3. Tratamiento de lixiviados mediante ozonización catalítica homogénea y heterogénea

\begin{tabular}{|c|c|c|c|c|c|}
\hline Fase del tratamiento & Catalizador & Parámetros iniciales & $\begin{array}{l}\text { Condiciones de } \\
\text { operación optimas }\end{array}$ & Eficacia & Referencia \\
\hline \multicolumn{6}{|c|}{ Homogénea } \\
\hline Postratamiento & $\mathrm{ZnSO}_{4}$ & $\begin{array}{l}\text { Efluente estabilizado } \\
\text { anaeróbicamente: } \\
4250 \mathrm{mg} / \mathrm{L} \text { de } \mathrm{DQO} \text {, } \\
348 \mathrm{mg} / \mathrm{L} \text { de } \mathrm{DBO}_{5} \text {, } \\
1897 \mathrm{mg} / \mathrm{L} \text { de } \mathrm{NH}_{3}-\mathrm{N} \text {, } \\
20300 \mathrm{Pt} \mathrm{Co} \text { de color, } \\
8.64 \mathrm{de} \mathrm{pH}\end{array}$ & $\begin{array}{l}27 \mathrm{~g} / \mathrm{m}^{3} \text { dosis de } \mathrm{O}_{3} \text {, } \\
1 \mathrm{~g} / 6 \mathrm{~g} \text { dosis de } \mathrm{Zn} \text {, } \\
4.0 \text { de } \mathrm{pH}, 180 \mathrm{~min} \text { de } \\
\text { reacción }\end{array}$ & $\begin{array}{l}90 \% \text { de DQO, } 99 \% \text { de } \\
\text { color, } 5 \% \text { de } \mathrm{NH}_{3}-\mathrm{N}\end{array}$ & [9] \\
\hline Postratamiento & $\mathrm{Fe}(\mathrm{III})$ & $\begin{array}{l}\text { Efluente biotratado: } \\
273 \text { de DQO., } 107.6 \\
\text { de TOC, } 8.58 \text { de, } 0.05 \\
\text { de BOD5/COD, } 3.71 \\
\mathrm{~cm}^{-1} \text { de absorbentes } \\
\mathrm{UV}_{254}\end{array}$ & $\begin{array}{l}100 \mathrm{mg} / \mathrm{min} \text { de } \mathrm{O}_{3}, 6 \\
\mathrm{~g} / \mathrm{L} \text { de } \mathrm{Fe}(\mathrm{III}), 6.5 \mathrm{de} \\
\mathrm{pH}, 25 \mathrm{~min} \text { de reacción }\end{array}$ & $\begin{array}{l}80.23 \% \text { de DQO y } \\
\text { el } 74.85 \% \text { de COT, } \\
85.01 \% \text { de absorbentes } \\
\mathrm{UV}_{254 .} \text {, reducción alta } \\
\text { de metales tóxicos }(\mathrm{Cu} \text {, } \\
\mathrm{Ni}, \mathrm{As}, \mathrm{Sr}, \mathrm{Cr}, \mathrm{Pb} \text { y } \mathrm{Zn})\end{array}$ & [41] \\
\hline \multicolumn{6}{|c|}{ Heterogénea } \\
\hline \multirow[t]{3}{*}{ Postratamiento } & $\mathrm{Al}_{2} \mathrm{O}_{3}$ & $\begin{array}{l}\text { Efluente de osmosis } \\
\text { inversa: } 1317.5 \mathrm{mg} / \mathrm{L} \\
\text { de DQO, } 20 \mathrm{mg} / \mathrm{L} \\
\text { de } \mathrm{DBO}_{5}, 57 \mathrm{mg} / \mathrm{L} \\
\text { de solidos suspendidos, } \\
7.3 \text { de pH, } 0.01 \mathrm{de} \\
\mathrm{BOD}_{5} / \mathrm{COD}\end{array}$ & $\begin{array}{l}22 \mathrm{mg} / \mathrm{min} \text {. de dosis de } \\
\mathrm{O}_{3}, 50 \mathrm{~g} / \mathrm{L} \text { de } \mathrm{Al}_{2} \mathrm{O}_{3}, \\
7.3 \text { de } \mathrm{pH}, 30^{\circ} \mathrm{C}, 30 \\
\text { min de reacción }\end{array}$ & $\begin{array}{llr}70 \% & \text { de } & \text { DQO, } \\
100 \% & \text { color, } & 0.2 \\
\text { de } \mathrm{DBO}_{5} / \mathrm{DQO} & \end{array}$ & [6] \\
\hline & $\begin{array}{l}\text { SSCWM/O }, \quad \text { Ti- } \\
\text { SSCWM/O }, \quad \text { Ti-Mn } \\
\text { /SSCWM/O } \\
\text { steel corrugated wire } \\
\text { mesh-SSCWM) }\end{array}$ & $\begin{array}{l}824.6 \pm 60 \mathrm{mg} / \mathrm{L} \text { de } \\
\mathrm{DQO}, 125.62 \pm 2 \\
\mathrm{mg} / \mathrm{L} \text { de } \mathrm{NH}_{3}-\mathrm{N}, 8.538 \\
\pm 0.002 \text { de } \mathrm{pH}, 350- \\
400 \text { de color, } 0.08 \mathrm{de} \\
\mathrm{BOD}_{5} / \mathrm{COD}\end{array}$ & $\begin{array}{l}8.5 \mathrm{de} \mathrm{pH}, 120 \mathrm{~min} \text { de } \\
\text { reacción. }\end{array}$ & $\begin{array}{l}64.44 \%, 68.97 \% \text { y } \\
78.43 \% \text { de DQO } \\
\text { para SSCWM/O3, } \\
\text { Ti-SSCWM/O3, Ti- } \\
\text { Mn /SSCWM/O3 } \\
\text { respectivamente; hasta } \\
\text { un 90\% de absorbentes } \\
\mathrm{UV}_{254}\end{array}$ & {$[25]$} \\
\hline & Zeolita neutral (faujasita) & $\begin{array}{l}2500 \mathrm{mg} / \mathrm{L} \text { de } \mathrm{DQO} \text {, } \\
40 \mathrm{mg} / \mathrm{L} \text { de } \mathrm{BOD}_{5} \text {, } \\
16200 \mathrm{PtCo} \text { de color, } \\
8.2 \mathrm{de} \mathrm{pH}, 1100 \mathrm{mg} / \mathrm{L} \\
\text { de } \mathrm{NO} 3-\mathrm{N}, 0.016 \mathrm{de} \\
\mathrm{BOD}_{5} / \mathrm{COD}\end{array}$ & $\begin{array}{l}27 \mathrm{~g} / \mathrm{Nm}^{3} \text { de dosis de } \\
\mathrm{O}_{3}, 11 \text { y } 5 \text { de } \mathrm{pH} \text { óp- } \\
\text { timos para reducción } \\
\text { de color y amoniaco } \\
\text { respectivamente, } 120 \\
\text { minutos }\end{array}$ & $\begin{array}{l}66 \% \text { de DQO, } 63 \% \\
\text { de Amoniaco, } 93 \% \\
\text { de color, } 0.1 \text { de } \\
\text { BOD }_{5} / \mathrm{COD}\end{array}$ & {$[32]$} \\
\hline \multirow[t]{2}{*}{ Postratamiento } & $\mathrm{Al}_{2} \mathrm{O}_{3}$ & $\begin{array}{l}\text { Efluente de un semi- } \\
\text { aerobic aged refuse } \\
\text { biofilter: } 1100 \mathrm{mg} / \mathrm{L} \\
\text { de DQO, } 3.0 \text { de ab- } \\
\text { sorbentes } \mathrm{UV}_{254}, 0.4 \\
\mathrm{~cm}^{-1} \text { de numero de } \\
\text { color }(\mathrm{CN}), 0.04 \text { de } \\
\mathrm{BOD}_{5} / \mathrm{COD}\end{array}$ & $\begin{array}{l}18.92 \mathrm{mg} / \mathrm{min} \text { de dosis } \\
\text { de } \mathrm{O}_{3}, \quad 10 \mathrm{~g} / \mathrm{L} \text { de } \\
\mathrm{Al}_{2} \mathrm{O}_{3}, 7.8 \pm 2 \mathrm{de} \mathrm{pH} \\
30 \text { min de reacción }\end{array}$ & $\begin{array}{l}54.3 \% \text { de DQO, } 82.9 \% \\
\text { de absorbentes } \mathrm{UV}_{254} \text {, } \\
95.9 \% \text { de } \mathrm{CN}\end{array}$ & {$[45]$} \\
\hline & $\begin{array}{l}\mathrm{Cu} / \mathrm{Mg} / \mathrm{Al} \text {-chitosan: } \\
\mathrm{Cu}(0.89 \mathrm{mmol} / \mathrm{L}), \mathrm{Mg} \\
(4 \mathrm{mmol} / \mathrm{L}) \text { y } \mathrm{Al}(2 \\
\mathrm{mmol} / \mathrm{L})\end{array}$ & $\begin{array}{l}40.70 \mathrm{mg} / \mathrm{L} \text { de } \mathrm{DQO}, \\
2100 \mathrm{mg} / \mathrm{L} \text { de } \mathrm{BOD}_{5} \\
9.1 \mathrm{de} \mathrm{pH}, 0.052 \mathrm{de} \\
\mathrm{BOD}_{5} / \mathrm{COD}, 34.04 \mathrm{de} \\
\text { COT }\end{array}$ & $\begin{array}{l}20 \mathrm{mg} / \mathrm{L} \text { de catalizador, } \\
3.5 \mathrm{mg} / \mathrm{min} \text { de } \mathrm{O}_{3}, 50 \\
\text { min de reacción }\end{array}$ & $\begin{array}{l}69 \% \text { de } \mathrm{DQO}, 48 \% \\
\text { de COT, } 54 \% \text { de ab- } \\
\text { sorbentes } \mathrm{UV}_{254}, 0.39 \\
\text { de } \mathrm{BOD}_{5} / \mathrm{COD}\end{array}$ & [46] \\
\hline
\end{tabular}

en conjunto, los residuos urbanos y los residuos industriales [54]. Por lo cual, se afirma que no existe un proceso de tratamiento único que funcione para todos los lixiviados [54].

Realizar un tratamiento eficiente de los lixiviados generados en los rellenos sanitarios de los países en desarrollo, se convierte en un proceso relevante de responsabilidad ambiental y social. Incluir los procesos basados en ozono junto con los tratamientos convencionales implementados en los rellenos sanitarios, debe depender de un proceso investigativo, donde se aporten recursos financieros, 
para lograr adaptar un acople de tratamiento adecuado, que optimice la gestión de los lixiviados cumpliendo con los estándares permisibles [54].

\section{Conclusiones}

La composición compleja de los lixiviados de rellenos sanitario requiere de una alternativa de tratamiento que alcance las remociones esperadas, de manera que no represente un riesgo para la salud y el ambiente. Recientemente, se han avanzado en estudios sobre los procesos basados en ozono para aprovechar sus ventajas oxidativas que degradan los compuestos recalcitrantes y mejoran la biodegradabilidad. Las técnicas de ozonización catalítica y peroxono son las más documentadas y desarrolladas como tratamiento. Se destaca la ozonización catalítica para remover altísimos porcentajes de DQO, COT, Color y compuestos absorbentes de $\mathrm{UV}_{254}$, y para disminuir el tiempo de reacción del tratamiento ( 25 a 180 minutos). Cada técnica presenta condiciones de operación distintas, de acuerdo a los tipos de reacciones que permiten la degradación de contaminantes. A pesar de los resultados prometedores de los procesos basados en ozono, para los países en desarrollo, continúa siendo un desafío para su implementación a escala real en un relleno sanitario, entre las causas principales, la limitación en cuanto a costos y de los sistemas de control.

\section{Referencias}

[1] M. Lovato, J. R. Buffelli, M. Abrile, and C. Martín, "Kinetics and efficiency of ozone for treatment of landfill leachate including the effect of previous microbiological treatment," Environ. Sci. Pollut. Res., vol. 26, no. 5, pp. 4474-4487, 2019.

[2] W. Cheng, X. Quan, X. Huang, C. Cheng, L. Yang, and Z. Cheng, "Enhancement of micro-filtration performance for biologicallytreated leachate from municipal solid waste by ozonation in a micro bubble reactor," Sep. Purif. Technol., vol. 207, pp. 535-542, 2018.

[3] S. Kow, M. R. Fahmi, C. Z. A. Abidin, and O. Soon-An, "Advanced Oxidation Processes:
Process Mechanisms, Affecting Parameters and Landfill Leachate Treatment," Water Environ. Res., vol. 88, no. 11, pp. 2047-2058, 2016.

[4] H. Wang, Y. nan Wang, X. Li, Y. Sun, $\mathrm{H}$. Wu, and D. Chen, "Removal of humic substances from reverse osmosis (RO) and nanofiltration (NF) concentrated leachate using continuously ozone generation-reaction treatment equipment," Waste Manag., vol. 56, pp. 271-279, 2016.

[5] A. Mojiri et al., "Concentrated landfill leachate treatment with a combined system including electro-ozonation and composite adsorbent augmented sequencing batch reactor process," Process Saf. Environ. Prot., vol. 111, pp. 253-262, 2017.

[6] Y. He, H. Zhang, J. J. Li, Y. Zhang, B. Lai, and Z. Pan, "Treatment of Landfill Leachate Reverse Osmosis Concentrate from by Catalytic Ozonation with $\gamma$-Al2O3," Environ. Eng. Sci., vol. 35, no. 5, pp. 501$511,2018$.

[7] Q. Xu, G. Siracusa, S. Di Gregorio, and Q. Yuan, "COD removal from biologically stabilized landfill leachate using Advanced Oxidation Processes (AOPs)," Process Saf. Environ. Prot., vol. 120, pp. 278-285, 2018.

[8] Z. Youcai, "Leachate Generation and Characteristics," in Pollution Control Technology for Leachate from Municipal Solid Waste, E. Inc., Ed. 2018, pp. 1-30.

[9] S. S. Abu Amr, H. A. Aziz, M. S. Hossain, and M. J. K. Bashir, "Simultaneous removal of COD and color from municipal landfill leachate using Ozone/Zinc sulphate oxidation process," Glob. Nest J., vol. 19, no. 3, pp. 498-504, 2017.

[10] W. Chen, A. Zhang, G. Jiang, and Q. Li, "Transformation and degradation mechanism of landfill leachates in a combined process of SAARB and ozonation," Waste Manag., vol. 85, pp. 283-294, 2019. 
[11] M. J. K. Bashir, H. A. Aziz, S. S. A. Amr, S. Sethupathi, C. A. Ng, and J. W. Lim, "The competency of various applied strategies in treating tropical municipal landfill leachate," Desalin. Water Treat., vol. 54, no. 9, pp. 23822395, 2015.

[12] H. Luo, Y. Zeng, Y. Cheng, D. He, and $\mathrm{X}$. Pan, "Recent advances in municipal landfill leachate: A review focusing on its characteristics, treatment, and toxicity assessment," Sci. Total Environ., vol. 703, p. 135468, 2020.

[13] W. Chen and Q. Li, "Elimination of UV-quenching substances from MBR- and SAARB-treated mature landfill leachates in an ozonation process: A comparative study," Chemosphere, vol. 242, p. 125256, 2020.

[14] Z. Huang, Z. Gu, Y. Wang, and A. Zhang, "Improved oxidation of refractory organics in concentrated leachate by a $\mathrm{Fe} 2+-$-enhanced O3/H2O2 process," Environ. Sci. Pollut. Res., vol. 26, no. 35, pp. 35797-35806, 2019.

[15] H. Qin and H. Chen, "Pretreatment of concentrated leachate by the combination of coagulation and catalytic ozonation with Ce/AC catalyst," Water Sci. Technol., vol. 73, no. 3, pp. 511-519, 2016.

[16] M. Han, X. Duan, G. Cao, S. Zhu, and S. H. Ho, "Graphitic nitride-catalyzed advanced oxidation processes (AOPs) for landfill leachate treatment: A mini review," Process Saf. Environ. Prot., vol. 139, pp. 230-240, 2020.

[17] M. Chys, V. A. Oloibiri, W. T. M. Audenaert, K. Demeestere, and S. W. H. Van Hulle, "Ozonation of biologically treated landfill leachate: Efficiency and insights in organic conversions," Chem. Eng. J., vol. 277, pp. 104-111, 2015.

[18] N. Amaral-Silva, R. C. Martins, S. CastroSilva, and R. M. Quinta-Ferreira, "Ozonation and perozonation on the biodegradability improvement of a landfill leachate," J.
Environ. Chem. Eng., vol. 4, no. 1, pp. 527533, 2016.

[19] J. L. Gao et al., "Integration of autotrophic nitrogen removal, ozonation and activated carbon filtration for treatment of landfill leachate," Chem. Eng. J., vol. 275, pp. 281287, 2015.

[20] J. Gao et al., "The present status of landfill leachate treatment and its development trend from a technological point of view," Rev. Environ. Sci. Biotechnol., vol. 14, no. 1, pp. 93-122, 2015.

[21] H.-J. Fan, Y.-Y. Chen, M.-Y. Chen, and D.T. Chang, Landfill leachate treatment with $\mathrm{Mn}$ and Ce oxides impregnated GAC-ozone treatment process, vol. 482. Elsevier B.V., 2015.

[22] R. Poblete, I. Oller, M. I. Maldonado, and E. Cortes, "Improved landfill leachate quality using ozone, UV solar radiation, hydrogen peroxide, persulfate and adsorption processes," J. Environ. Manage., vol. 232, no. August 2018, pp. 45-51, 2019.

[23] K. Ikehata and Y. Li, "Ozone-Based Processes," in Advanced Oxidation Processes for Wastewater Treatment: Emerging Green Chemical Technology, Elsevier Inc., Ed. 2018, pp. 115-134.

[24] H. Wang et al., "Transformation of dissolved organic matter in concentrated leachate from nanofiltration during ozone-based oxidation processes (O3, O3/H2O2 and O3/UV)," J. Environ. Manage., vol. 191, pp. 244-251, 2017.

[25] A. Soubh and N. Mokhtarani, "The post treatment of composting leachate with a combination of ozone and persulfate oxidation processes," RSC Adv., vol. 6, no. 80, pp. 76113-76122, 2016.

[26] S. S. Abu-Amr, S. N. F. Zakaria, and H. A. Aziz, "Performance of combined ozone and zirconium tetrachloride in stabilized landfill 
leachate treatment," J. Mater. Cycles Waste Manag., vol. 19, no. 4, pp. 1384-1390, 2016.

[27] A. P. J. Scandelai, L. Cardozo Filho, D. C. C. Martins, T. K. F. de S. Freitas, J. C. Garcia, and C. R. G. Tavares, "Combined processes of ozonation and supercritical water oxidation for landfill leachate degradation," Waste Manag., vol. 77, no. November, pp. 466-476, 2018.

[28] E. Kattel, A. Kivi, K. Klein, T. Tenno, N. Dulova, and M. Trapido, "Hazardous waste landfill leachate treatment by combined chemical and biological techniques," Desalin. Water Treat., vol. 57, no. 28, pp. 13236-13245, 2016.

[29] A. I. Gomes, T. F. Soares, T. F. C. V. Silva, R. A. R. Boaventura, and V. J. P. Vilar, "Ozone-driven processes for mature urban landfill leachate treatment: Organic matter degradation, biodegradability enhancement and treatment costs for different reactors configuration," Sci. Total Environ., vol. 724, 2020.

[30] J. Umamaheswari, T. Bharathkumar, S. Shanthakumar, and K. M. Gothandam, "A feasibility study on optimization of combined advanced oxidation processes for municipal solid waste leachate treatment," Process Saf. Environ. Prot., vol. 143, pp. 212-221, 2020.

[31] F. Jiang, B. Qiu, and D. Sun, "Degradation of refractory organics from biologically treated incineration leachate by VUV/O3," Chem. Eng. J., vol. 370, no. December 2018, pp. 346$353,2019$.

[32] H. R. AlGburi, H. A. Aziz, H. M. Zwain, and A. F. M. Noor, "Treatment of Landfill Leachate by Heterogeneous Catalytic Ozonation with Granular Faujasite Zeolite," Environ. Eng. Sci., vol. 00, no. 00, 2020.

[33] M. Bourgin et al., "Effect of operational and water quality parameters on conventional ozonation and the advanced oxidation process
$\mathrm{O} 3 / \mathrm{H} 2 \mathrm{O} 2$ : Kinetics of micropollutant abatement, transformation product and bromate formation in a surface water," Water Res., vol. 122, pp. 234-245, 2017.

[34] T. H. Van and T. V. Trinh, "Application of Regression Analysis for Ozone and Catalytic Ozonation of Organic Compounds from Landfill Leachate with Ceramic Raschig Rings and Natural Manganese Ore," Ozone Sci. Eng., vol. 38, no. 2, pp. 133-142, 2016.

[35] P. Asaithambi, B. Sajjadi, A. R. Abdul Aziz, and W. M. A. B. W. Daud, "Ozone (O3) and sono (US) based advanced oxidation processes for the removal of color, COD and determination of electrical energy from landfill leachate," Sep. Purif. Technol., vol. 172, pp. 442-449, 2017.

[36] F. Wang, Y. Huang, P. Wen, and Q. Li, "Transformation mechanisms of refractory organic matter in mature landfill leachate treated using an $\mathrm{Fe} 0$-participated $\mathrm{O} 3 / \mathrm{H} 2 \mathrm{O} 2$ process," Chemosphere, vol. 263, p. 128198, 2021.

[37] P. Gautam, S. Kumar, and S. Lokhandwala, "Advanced oxidation processes for treatment of leachate from hazardous waste landfill: A critical review," J. Clean. Prod., vol. 237, 2019.

[38] C. Wu, W. Chen, Z. Gu, and Q. Li, "A review of the characteristics of Fenton and ozonation systems in landfill leachate treatment," Sci. Total Environ., p. 143131, 2020.

[39] M. Ghahrchi and A. Rezaee, "Electrocatalytic ozonation for improving the biodegradability of mature landfill leachate," J. Environ. Manage., vol. 254, no. October 2019, p. 109811, 2020.

[40] A. P. J. Scandelai, E. Sloboda Rigobello, B. L. C. de Oliveira, and C. R. G. Tavares, "Identification of organic compounds in landfill leachate treated by advanced oxidation processes," Environ. Technol. 
(United Kingdom), vol. 40, no. 6, pp. 730741, 2019.

[41] Y. Wang et al., "Effective removal of contaminants from biotreated leachate by a combined $\mathrm{Fe}(\mathrm{III}) / \mathrm{O} 3$ process: Efficiency and mechanisms," J. Clean. Prod., vol. 276, no. Iii, p. 123379, 2020.

[42] Z. Liu, L. Pan, F. Hu, and Y. Hu, "Advanced landfill leachate biochemical effluent treatment using Fe-Mn/AC activates O3/Na2S2O8 process: process optimization, wastewater quality analysis, and activator characterization," Environ. Sci. Pollut. Res., vol. 27, no. 13, pp. 15337-15349, 2020.

[43] H. R. AlGburi, H. A. Aziz, H. M. Zwain, and A. F. M. Noor, "Treatment of Landfill Leachate by Heterogeneous Catalytic Ozonation with Granular Faujasite Zeolite," Environ. Eng. Sci., no. December, 2020.

[44] A. Ikhlaq et al., " Treatment of leachate through constructed wetlands using Typha angustifolia in combination with catalytic ozonation on Fe-zeolite A , Int. J. Phytoremediation, vol. 0, no. 0, pp. 1-9, 2020.

[45] Y. Xiang, Y. Chen, S. Luo, J. Zou, and A. Zhang, "Degradation of recalcitrant organic matter in SAARB leachate by a combined process of coagulation and catalytic ozonation," Environ. Sci. Pollut. Res., vol. 27, no. 32, pp. 40219-40228, 2020.

[46] D. Ranjbar-Vakilabadi, A. H. Hassani, G. Omrani, and B. Ramavandi, "Catalytic potential of $\mathrm{Cu} / \mathrm{Mg} / \mathrm{Al}$-chitosan for ozonation of real landfill leachate," Process Saf. Environ. Prot., vol. 107, pp. 227-237, 2017.

[47] M. A. Kamaruddin, M. S. Yusoff, L. M. Rui, A. M. Isa, M. H. Zawawi, and R. Alrozi, "An overview of municipal solid waste management and landfill leachate treatment: Malaysia and Asian perspectives," Environ.
Sci. Pollut. Res., vol. 24, no. 35, pp. $26988-$ 27020, 2017.

[48] R. A. Agarwal, A. K. Agarwal, T. Gupta, and N. Sharma, Introduction to Pollutants from Energy Sources: Characterization and Control. 2019.

[49] Y. Ogata et al., "Design considerations of constructed wetlands to reduce landfill leachate contamination in tropical regions," J. Mater. Cycles Waste Manag., vol. 20, no. 4, pp. 1961-1968, 2018.

[50] C. Ma et al., "Catalytic micro-ozonation by Fe3O4 nanoparticles@ cow-dung ash for advanced treatment of biologically pre-treated leachate," Waste Manag., vol. 83, pp. 23-32, 2019.

[51] X. Tang, S. Zhu, X. Quan, X. Huang, F. Qiu, and C. Feng, "Degradation Treatment of Concentrated Landfill Leachate by Catalytic Ozonation in a Microbubble Reactor," Ozone Sci. Eng., vol. 00, no. 00, pp. 1-14, 2020.

[52] K. S. Rajoo, D. S. Karam, A. Ismail, and A. Arifin, "Evaluating the leachate contamination impact of landfills and open dumpsites from developing countries using the proposed Leachate Pollution Index for Developing Countries (LPIDC)," Environ. Nanotechnology, Monit. Manag., vol. 14, no. September, p. 100372, 2020.

[53] W. L. M. Braga et al., "Optimization of the treatment of sanitary landfill by the ozonization catalysed by modified nanovermiculite in a rotating packed bed," J. Clean. Prod., vol. 249, p. 119395, 2020.

[54] S. M. Iskander et al., "A review of landfill leachate induced ultraviolet quenching substances: Sources, characteristics, and treatment," Water Res., vol. 145, pp. 297-311, 2018. 